\title{
The Diaphragm Pump Spindle Fault Diagnosis Using HHT Based on EMD
}

\author{
Yin Jia ${ }^{*}, 1$ Li Qingmao ${ }^{1}$ and Yuan Xuyi ${ }^{2}$ \\ ${ }^{I}$ School of Physical and Electronic Science, Aba Teachers College, Wenchuan, Sichuan 623002, P.R. China \\ ${ }^{2}$ School of Information Engineering and Automation, Kunming University of Science and Technology, Kunming, Yunnan \\ 650500, P.R. China
}

\begin{abstract}
The running performance of reciprocating piston diaphragm pump spindle affects reciprocating diaphragm pump operating conditions directly, even the effective operation of the entire pipeline transportation systems. Because of the diversity and uncertainty of the diaphragm pump spindle fault, this paper proposes a fault diagnosis method of HHT based on EMD. Firstly, it can process the original signal vibration data by EMD to get some IMF, then analyze each IMF through Hilbert transform to get HHT marginal spectrum, finally study the HHT marginal spectrum of IMF to obtain equipment fault diagnosis and research. The experimental results show that the method of HHT based on EMD is effective and feasible for fault diagnosis of reciprocating piston diaphragm pump spindle.
\end{abstract}

Keywords: EMD, failure diagnosis, HHT, marginal spectrum, reciprocating piston diaphragm pump, spindle.

\section{INTRODUCTION}

With the continuous development of pipeline technology, the pipeline transport is not only bringing considerable economic value, but also changing the ways the people live. Reciprocating piston diaphragm pump which is the power output device pipeline is one of the core equipments of pipeline system, so the security issue of reciprocating piston diaphragm pump is the key issue of pipeline transport. The spindle is one of the most important parts of a reciprocating piston diaphragm pump. In the process of diaphragm pump operation, the spindle failure is most deadly, i.e. once the spindle appears faulty, it will cause huge economic losses, and even casualties. Therefore, the fault diagnosis of reciprocating diaphragm pump spindle is importantly and valuably.

Due to the failures of the reciprocating piston diaphragm pump such as diversity, uncertainty and concurrency [1,2], fault diagnosis of diaphragm spindle is very difficult. This paper presents a method of Hilbert-Huang Transform (HHT) based on EMD for reciprocating piston diaphragm pump spindle fault diagnosis. Firstly, it can process original vibration signal by empirical mode decomposition EMD to get a limited number of intrinsic mode function IMF; then it analyzed each IMF through Hilbert transform to get HHT marginal spectrum; finally studies the HHT marginal spectrum of IMF for further research. Thus, HHT based method can accurately reflect the failure frequency characteristic of reciprocating piston diaphragm pump spindle through analyzing the strong change fault characteristics of IMF marginal spectrum.

*Address correspondence to this author at the School of Physical and Electronic Science, Aba Teachers College, Wenchuan, Sichuan 623002, P.R. China; Tel: +86 18011262838; E-mail: deathcity@sohu.com

\section{RECIPROCATING DIAPHRAGM AND SPINDLE INTRODUCTION}

Reciprocating piston diaphragm pump is mainly composed of motor, crankshaft, connecting rod, slider, spindle, piston and so on. It generates power by motor to drive crankshaft in a circular motion, then the connecting rod drives spindle reciprocating movement together, so the spindle transmits power to piston to squeeze the oil medium, which produces difference in liquid pressure by the oil medium to complete the slurry transportation [3].

\section{EMD AND HHT}

HHT has been prepared and adapted to non-linear signal or non-stationary signal. The complex signal can be decomposed into a finite number of intrinsic mode function IMF by EMD. It uses Hilbert transform to obtain the instantaneous frequency and instantaneous amplitude on each IMF, and then it obtains Hilbert spectrum and marginal spectrum of the signal, to achieve fault diagnostic analysis [4].

\subsection{EMD}

The EMD method is a process by which IMF can be separated from complex signal. The instantaneous frequency of complex signal after Hilbert transform has a physical meaning $[5,6]$. EMD decomposition process is summarized below:

(1) Determine all local extreme points of the signal $x(t)$

(2) Connect all the local maximum points and minimum points respectively, leading to formation of one upper envelope and one lower envelope.

(3) Denote the average of the upper envelope and lower envelope as $m(t)$, is given by: 


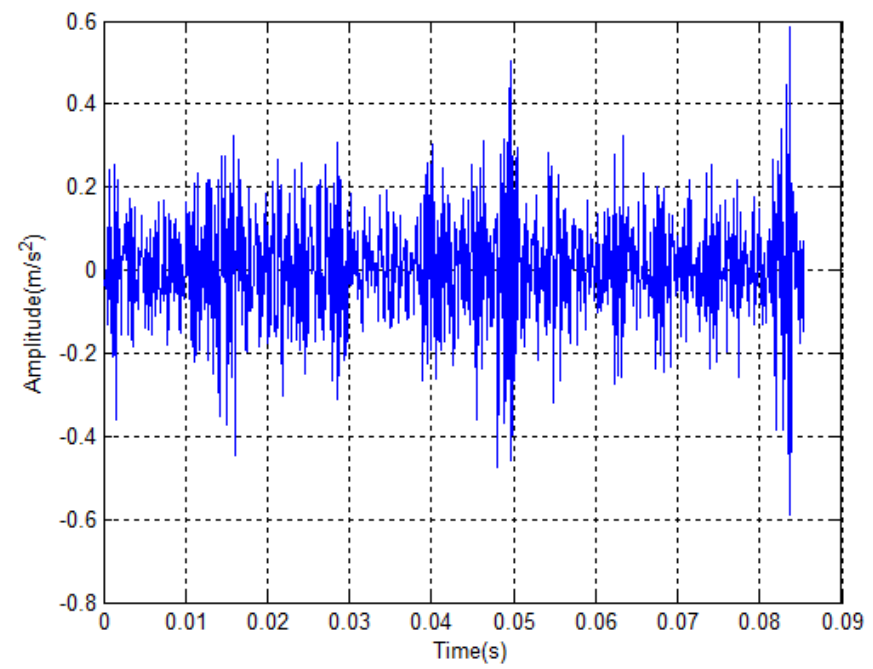

Fig. (1). Original abnormal vibration signal.

$c(t)=x(t)-m(t)$

Check, if $c(t)$ is an IMF, then $c(t)$ is the first IMF of $x(t)$, so a residual function can be obtained through separating $c(t)$ from $x(t)$.

$r(t)=x(t)-c(t)$

Otherwise, the $c(t)$ as original data, repeat steps (1) - (3), until $r(t)$ becomes a monotonic function that can't extract any IMF, the cycle ends. Complete the EMD decomposition.

In this way, the original signal can be expressed as the sum of a residual function and some IMFs:

$x(t)=\sum_{i=1}^{n} c_{i}(t)+r_{n}(t)$

\subsection{HHT and Marginal Spectrum}

Hilbert transforms the IMFs to calculate the instantaneous frequency. First, it can transform all IMF [7], namely:

$H\left[c_{i}(t)\right]=\frac{1}{\pi} \int_{-\infty}^{\infty} \frac{c_{i}(t)}{t-\tau} d \tau$

Structure analysis signal to obtain amplitude and phase function:

$a_{i}(t)=\sqrt{c_{i}^{2}(t)+\left(H\left[c_{i}(t)\right]\right)^{2}}$

$\Phi_{i}=\arctan \frac{H\left[c_{i}(t)\right]}{c_{i}(t)}$

The instantaneous frequency is:

$w_{i}(t)=\frac{d \Phi_{i}(t)}{d t}$

So
$x(t)=H(w, t)=\operatorname{Re} \sum_{i=1}^{n} a_{i}(t) \exp \left(j \int w_{i}(t) d t\right)$

Here omit residual amount $r_{n}(t)$, Re represents the real component, and $H(w, t)$ becomes known as the Hilbert spectrum. Further, marginal spectrum is defined as follows:

$h(w)=\int_{0}^{T} H(w, t) d t$

Here, $\mathrm{T}$ is the total length of signal. The instantaneous frequency and amplitude can be described as the time and frequency of the original signal. The Hilbert spectrum accurately describes the amplitude of the signal on the frequency changing with time and frequency, and $h(w)$ reflects the signal amplitude with the change of frequency in the whole frequency [8].

\section{EXPERIMENTAL SIMULATION}

In this paper, the vibration signal data from the USA Case Western Reserve University is adopted as the experimental simulation data, in order to try simulation and verification to prove the feasibility and effectiveness of the method. At the bearing rotating speed of $1797 \mathrm{r} / \mathrm{min}$, the sampling frequency is $12 \mathrm{kHz}$, with the use of 1024 points. Fig. (1) shows the original abnormal vibration signal; Fig. (2) shows the original normal vibration signal. Fig. (1) and Fig. (2) show that the frequency characteristics can be obtained by significant changes occurred in the vibration amplitude. It indicates that although the device does show abnormal situation, yet it cannot determine the fault feature. So it needs for further analysis and processing for the original signal.

In order to further analyze and research the vibration signal, it can obtain the corresponding vibration signal spectrum from the original signal on EMD decomposition; decomposition process is shown in Fig. (3). Fig. (3) shows IMFs of abnormal vibration signal after EMD decomposition. It can be seen from Fig. (3), that EMD decomposition is a vibration signal decomposition process moves gradually from high 


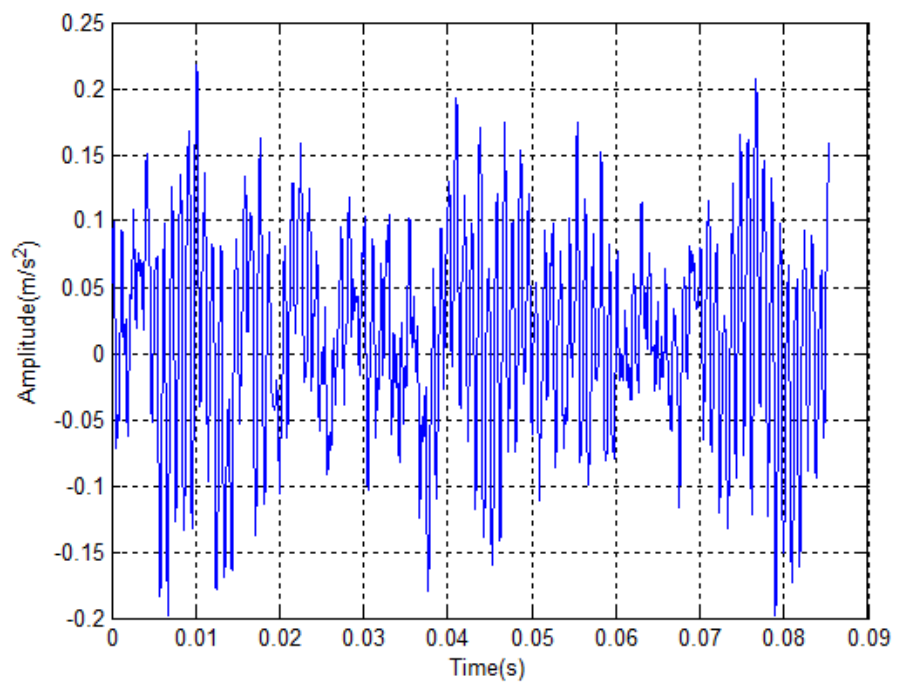

Fig. (2). Original normal vibration signal.

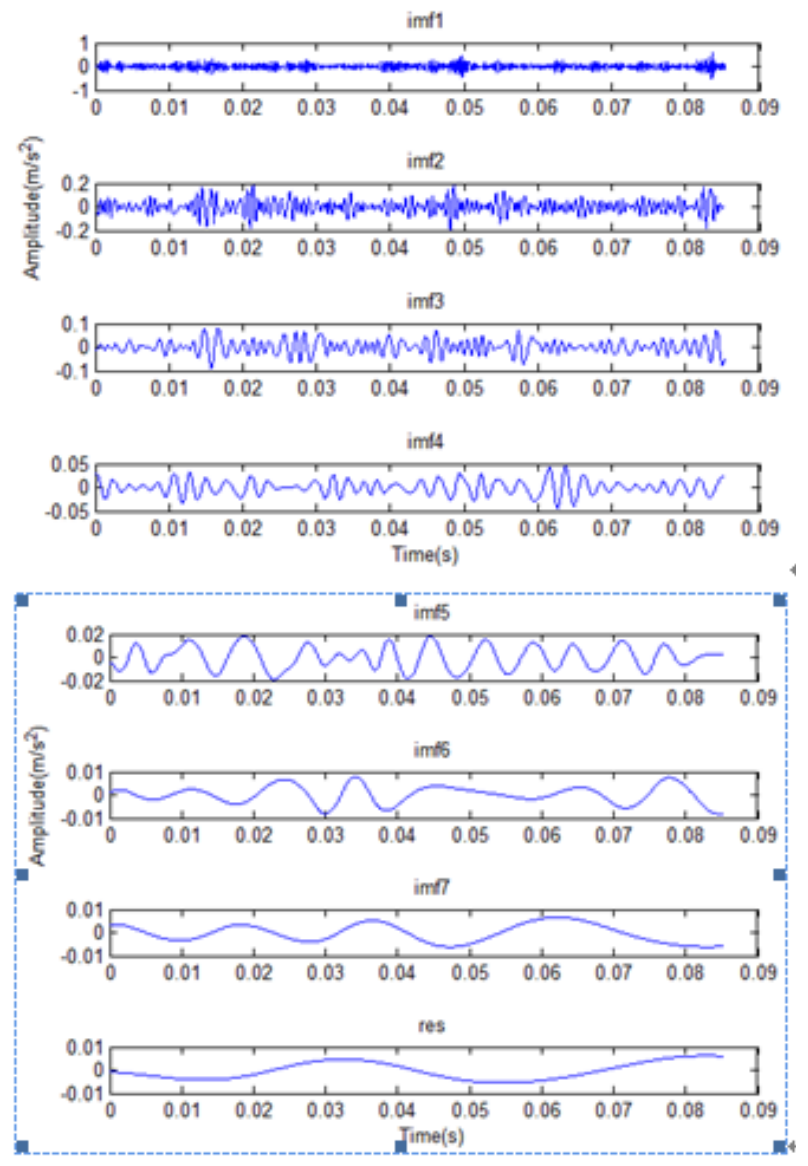

Fig. (3). IMFs of abnormal vibration signal after EMD decomposition.

frequency to low frequency and an abnormal vibration signal is reasonably decomposed into 7 IMFs and a residual amount Res. The frequency of each IMF gradually reduces from the top to bottom. Each IMF includes a unique band frequency characteristic, which is the characteristic frequency of the vibration signal failure that provides a good basis for fault diagnosis of research. Here, it can abandon the residual amount of Res.

In the process of equipment fault diagnosis research, the characteristics of each IMF are studied to analyze the targeted equipment running status, so that it can achieve fault 

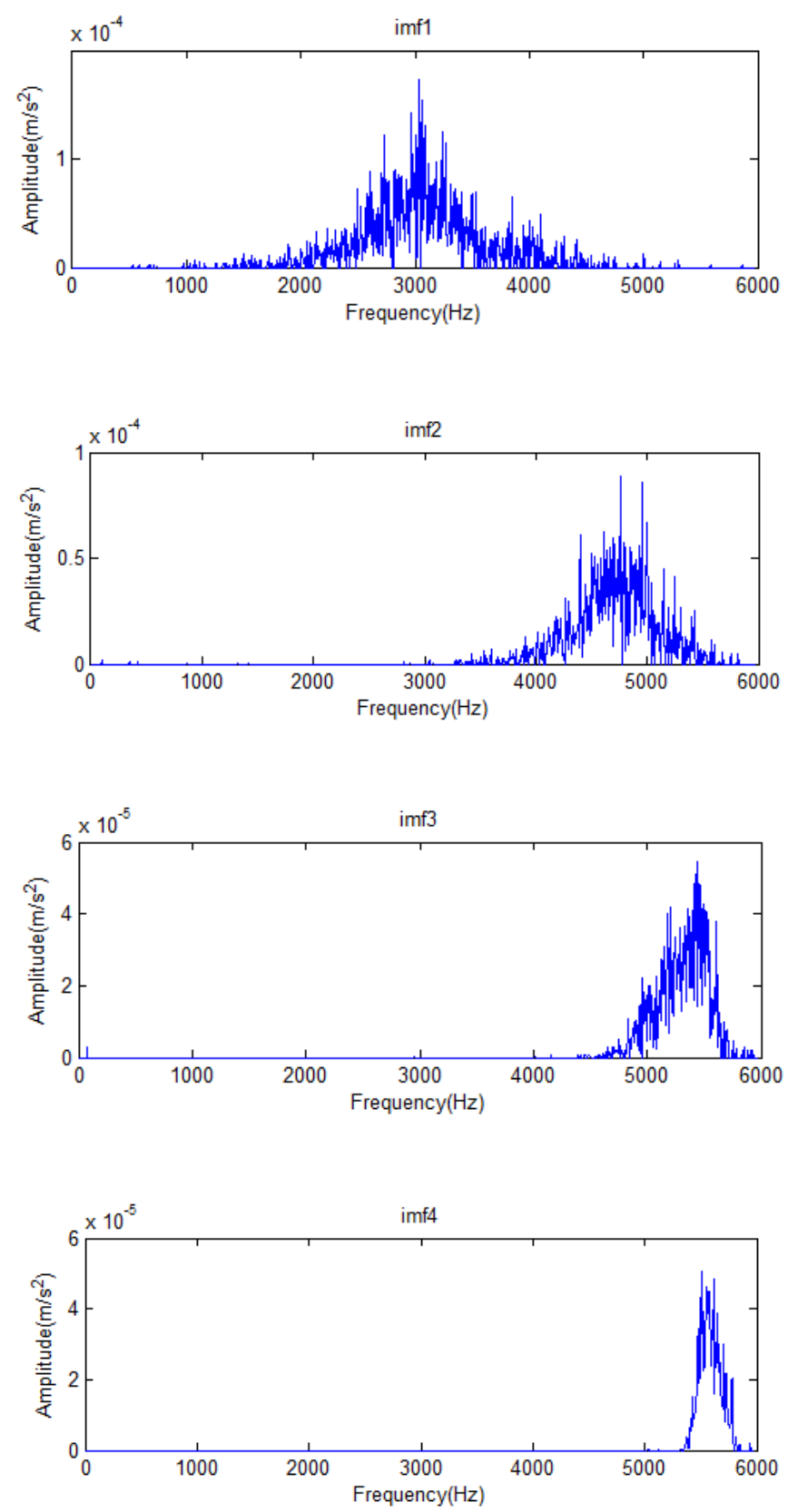

Fig. (4). IMF1-IMF4 marginal spectrum of abnormal vibration signal.

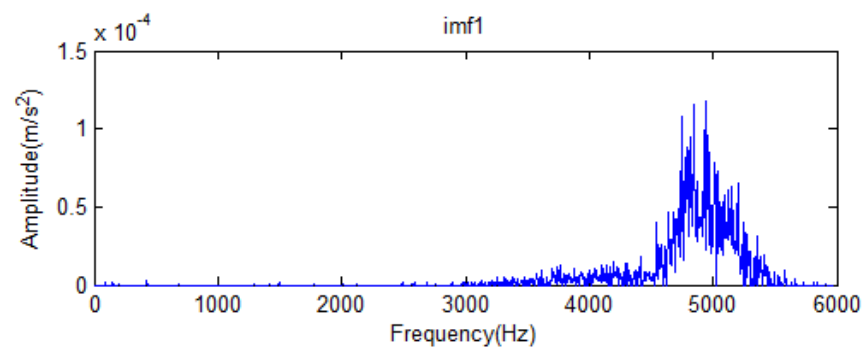

Fig. (5). Contd... 

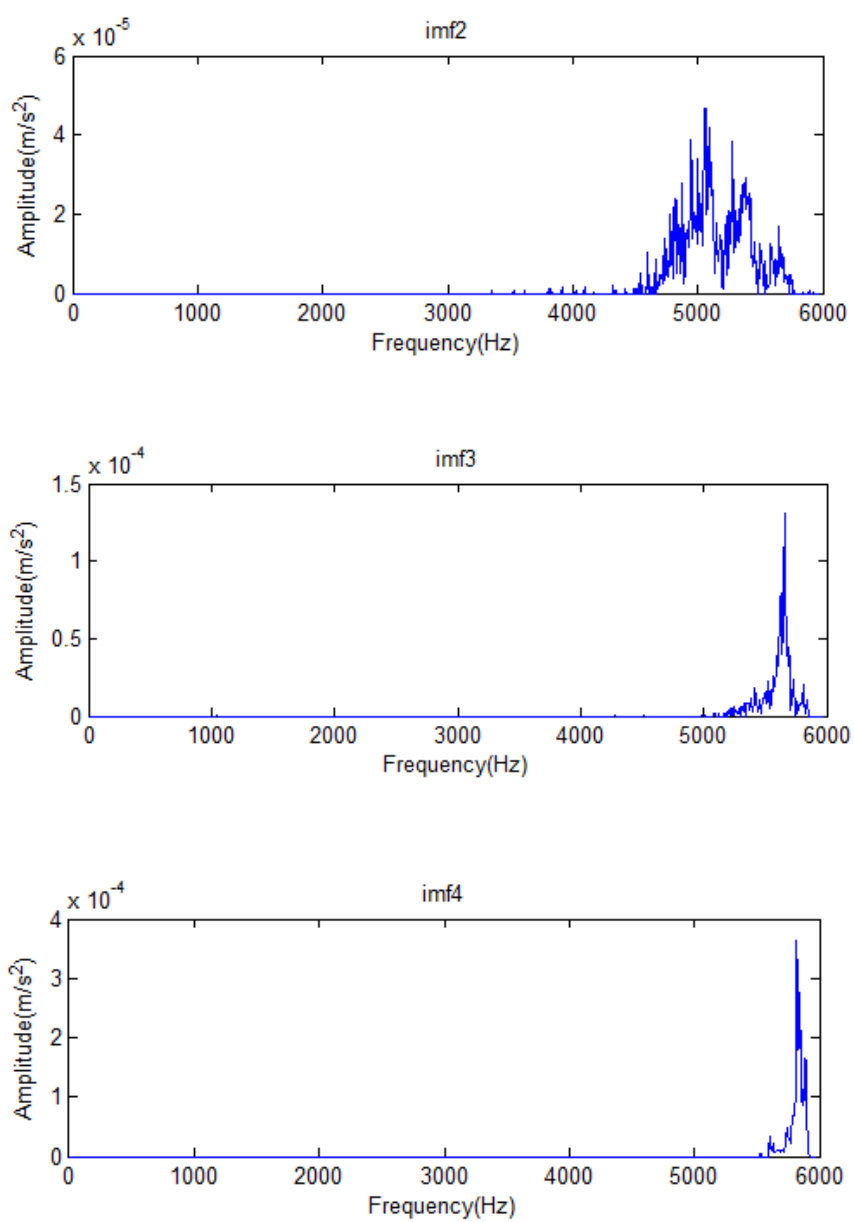

Fig. (5). IMF1-IMF4 marginal spectrum of normal vibration signal.

diagnosis. The changes in IMF1-IMF4 characteristics are very obvious, which contain rich vibration information. Therefore, this article takes IMF1-IMF4 as the main study object to analyze their characteristics for fault diagnosis research and analysis.

Make use of Hilbert transform to deal with each IMF, which can get the instantaneous frequency and instantaneous amplitude of each IMF to obtain signal's Hilbert marginal spectrum, so as to realize the fault diagnosis. Whereas Fig. (4) shows IMF1-IMF4 marginal spectrum of abnormal vibration signal, Fig. (5) shows IMF1-IMF4 marginal spectrum of normal vibration signal.

By comparing and analyzing Fig. (4) and Fig. (5), it is obvious that the IMF1 vibration frequency of normal vibration signal focuses at $4500 \mathrm{~Hz}$ to $5500 \mathrm{~Hz}$, while the IMF1 vibration frequency of abnormal vibration signal emerges a large number of abnormal vibration signals at $2000 \mathrm{~Hz}$ to $4500 \mathrm{~Hz}$; and a peak appears at $3000 \mathrm{~Hz}$. The surrounding is accompanied by the emergence of the frequency doubling and frequency division, which provides rich vibration information and powerful energy, so it is worthy of further research and analysis.

The IMF2 vibration frequency of normal vibration signal mainly focuses at $4500 \mathrm{~Hz}$ to $5800 \mathrm{~Hz}$, while the IMF2 vibration frequency of abnormal vibration signal mainly focuses at $4000 \mathrm{~Hz}$ to $5500 \mathrm{~Hz}$. So it emerges a large number of abnormal vibration signals, and the amplitude is significantly increased. Visibly, the frequency characteristics of the abnormal vibration signal change, especially the peak shifts from near $5000 \mathrm{~Hz}$ to near $4800 \mathrm{~Hz}$, and it generates a lot of vibration energy information.

The IMF3 vibration frequency of normal vibration signal mainly focuses on $5200 \mathrm{~Hz}$ to $5800 \mathrm{~Hz}$, which is a single peak and clear waveform, while the IMF3 vibration frequency of abnormal vibration signal focuses on $4800 \mathrm{~Hz}$ to $5800 \mathrm{~Hz}$, and there is a large number of abnormal vibration signals. It contains a lot of vibration feature information, and the amplitude is also significantly increased. The IMF4 frequency of normal vibration signal and abnormal vibration signal concentrates at $5000 \mathrm{~Hz}$ to $6000 \mathrm{~Hz}$, with the increased amplitude.

Visibly, IMF1-IMF4 marginal spectrum of equipment has significant changes, which is the equipment problem for which, equipment management personnel need to be targeted for fault diagnosis and analysis. By comparing and studying the changes in each IMF marginal spectrum, it can find the changes in characteristic frequency of equipment to locate the equipment fault position and improve diagnosis, so as to avoid blind fault diagnosis. But determining the 
equipment failure need to further study the specific frequency characteristics responsible for the equipment failure.

\section{CONCLUSION}

The EMD has strong ability for processing unsteady and nonlinear signal. The simulation results show the fault diagnosis of reciprocating piston diaphragm pump spindle, using the HHT based on EMD method, is effective and feasible. Through the analysis of marginal spectrum, it can accurately get the diaphragm pump spindle running status. Therefore, the HHT method based on EMD for fault diagnosis of reciprocating diaphragm pump spindle is of great significance.

\section{CONFLICT OF INTEREST}

The authors confirm that this article content has no conflict of interest.

\section{ACKNOWLEDGEMENTS}

This work was financially supported by the foundation items: Science \& Research Program of Sichuan province (No. 14ZB0339 \& No. 15ZB0351); Science \& Research Program of Aba Teachers College (No. ASC14-27 \& No. ASC14-30).

\section{REFERENCES}

[1] L. Wang, J. Liu, and S.Y. Liu, "Analysis on dynamic characteristic of crankshaft of reciprocating piston diaphragm pump", Machinery Design and Manufacture, vol. 5, pp. 238-239, 2010.

[2] C. Jiang, "Study on Failure Diagnosis System of the Large-scale Diaphragm Pump Group", M.S. thesis, Northeastern University, Liaoning, China, 2008.

[3] J. Yin, J.D. Wu, and X.D. Wang, "The reciprocating diaphragm pump spindle fault diagnosis based on HHT", Transducer and Microsystem Technologies, vol. 32, no. 4, pp. 5-8, 2013.

[4] J.J. Wu, "Pump fault diagnosis based on self-adaptive wavelet denoise", Control and Instruments in Chemical Industry, vol. 37, no. 4 , pp. 36-38, 2010

[5] N.E. Huang, Z. Shen, S. R. Long, M. C. Wu, H. H. Shih, Q. Zheng, N. C. Yen, C. C. Tung, and H. H. Liu, "The empirical mode decomposition and the Hilbert spectrum for nonlinear and nonstationary time series analysis", Proceedings of the Royal Society of London A. London: The Royal Society, vol. 454, pp. 903- 995, 1998.

[6] X.N. Zhang, Q.S. Zeng, and H. Wang, "Bearing fault diagnosis based on improved wavelet denoising and EMD method", Measurement \& Control Technology, vol. 33, no. 1, pp. 23-26, 2014.

[7] Q.K. Han, and X.G. Yu, Principle and Application of Modern Machinery Fault Diagnosis Based on Vibration Analysis, Beijing, 2010, pp. 81-82.

[8] Z.J. He, Mechanical Fault Diagnosis Theory and Application, Beijing, 2010, pp. 74-81.

Received: May 16, 2015

(C) Jia et al.; Licensee Bentham Open.

This is an open access article licensed under the terms of the Creative Commons Attribution Non-Commercial License (http://creativecommons.org/licenses/by$\mathrm{nc} / 4.0 /$ ) which permits unrestricted, non-commercial use, distribution and reproduction in any medium, provided the work is properly cited. 